\title{
Hyperbolic Cosines and Sines Theorems for the Triangle Formed by Arcs of Intersecting Semicircles on Euclidean Plane
}

\author{
Robert M. Yamaleev ${ }^{1,2}$ \\ ${ }^{1}$ Joint Institute for Nuclear Research, Laboratory of Information Technology, Street Joliot Curie 6, \\ CP 141980, P.O. Box 141980, Dubna, Russia \\ ${ }^{2}$ Universidad Nacional Autonoma de Mexico, Facultad de Estudios Superiores, Avenida 1 Mayo, \\ 54740 Cuautitlán Izcalli, MEX, Mexico \\ Correspondence should be addressed to Robert M. Yamaleev; iamaleev@servidor.unam.mx
}

Received 17 December 2012; Accepted 21 February 2013

Academic Editor: Sujit Samanta

Copyright (C) 2013 Robert M. Yamaleev. This is an open access article distributed under the Creative Commons Attribution License, which permits unrestricted use, distribution, and reproduction in any medium, provided the original work is properly cited.

\begin{abstract}
The hyperbolic cosines and sines theorems for the curvilinear triangle bounded by circular arcs of three intersecting circles are formulated and proved by using the general complex calculus. The method is based on a key formula establishing a relationship between exponential function and the cross-ratio. The proofs are carried out on Euclidean plane.
\end{abstract}

\section{Introduction}

Classically, the models of the hyperbolic plane are regarded as based on Euclidean geometry. One starts with a piece of a Euclidean plane, a half-plane, or a circular disk and then, in that half-plane or disk the notions of points, lines, distances, angles are defined as things that could be described in terms of Euclidean geometry [1-3]. The model of the hyperbolic plane is the half-plane model. The underlying space of this model is the upper half-plane model $H$ in the complex plane $C$, defined to be

$$
H=\{z \in C: \operatorname{Im}(z)>0\} .
$$

In coordinates $(x, y)$ the line element is defined as

$$
d s^{2}=\frac{1}{y^{2}}\left(d x^{2}+d y^{2}\right) .
$$

The geodesics of this space are semicircles centered on the $x$ axis and vertical half-lines. The geometrical properties of the figures on the half-plane are studied by considering quantities invariant under an action of the general Möbius group, which consists of compositions of Möbius transformations and reflections [4]. The curvilinear triangle formed by circular arcs of three intersecting semicircles is one of the principal figures of the upper half-plane model $H$. The hyperbolic laws of sines-cosines for that triangle are proved by using properties of the Möbius group and the upper half-plane $H$.

In this paper we suggest another way of construction of proofs of the sines-cosines theorems of the Poincaré model. The curvilinear triangle formed by circular arcs is the figure of the Euclidean plane; consequently, on the Euclidean plane we have to find relationships antecedent to the sines-cosines hyperbolic laws. Therefore, first of all, we establish these relationships by making use of axioms of the Euclidean plane, only. Secondly, we proove that these relationships can be formulated as the hyperbolic sine-cosine theorems. For that purpose we refer to the general complex calculus and within its framework establish a relationship between exponential function and the cross-ratio. In this way the hyperbolic trigonometry emerges on Euclidean plane in a natural way.

The paper is organized as follows. In Section 2 a key formula connecting the hyperbolic calculus with the crossratio is established. By employing the key formula and the Pythagoras theorem the elements of the right-angled triangle are expressed as functions of the hyperbolic trigonometry. In Section 3 we explore Euclidian properties of the curvilinear triangle bounded by circular arcs of three intersecting semicircles. Two main relationships connecting three intersecting semicircles are established. In Section 4, we prove the theorem of cosines for the curvilinear triangle bounded by the 


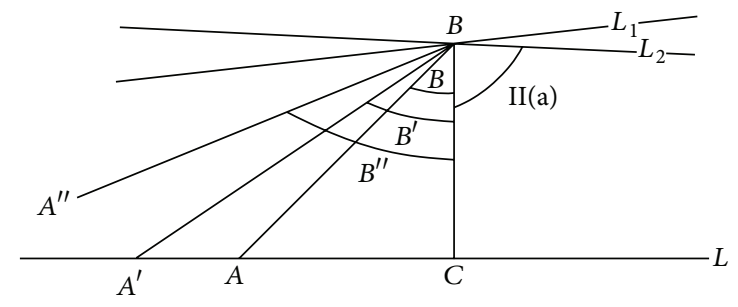

FIGURE 1: Motion of the hypothenuse of the right angled triangle.

circular arcs. In Section 5, the hyperbolic law of sines and second hyperbolic law of cosines are derived on the basis of the main relationships between these semicircles.

\section{Hyperbolic Trigonometry in Euclidean Geometry}

2.1. Trigonometry Induced by General Complex Algebra. The simplest generalization of the complex algebra, denominated as General Complex Algebra, is defined by unique generator $\mathbf{e}$ satisfying the quadratic equation [5]

$$
\mathbf{e}^{2}-b_{1} \mathbf{e}+b_{0}=0
$$

where coefficients $b_{0}, b_{1}$ are given by real positive numbers and $b_{1}^{2}-4 b_{0}>0$.

The following Euler formula holds true:

$$
\exp (\mathbf{e} \phi)=g_{0}(\phi)+\mathbf{e} g_{1}(\phi)
$$

Denote by $x_{1}, x_{2} \in R$ roots of the quadratic equation (3). The Euler formula (4) is decomposed into two independent equations:

$$
\exp \left(x_{k} \phi\right)=g_{0}(\phi)+x_{k} g_{1}(\phi), \quad k=1,2,
$$

from which one may find explicit formulae for $g$-functions. These functions are linear combinations of the exponential functions $\exp \left(x_{k} \phi\right), k=1,2$. Geometrical interpretation of the general complex algebra is done in [6].

Form the following ratio

$$
\exp \left(\left(x_{2}-x_{1}\right) \phi\right)=\frac{x_{2}-D(\phi)}{x_{1}-D(\phi)}
$$

where

$$
D(\phi)=-\frac{g_{0}(\phi)}{g_{1}(\phi)} .
$$

Introduce a pair of variables $X_{1}, X_{2}$ by

$$
X_{k}(\phi)=x_{k}-D(\phi), \quad k=1,2 .
$$

Then, (6) is written as follows:

$$
\exp \left(\left(X_{2}-X_{1}\right) \phi\right)=\frac{X_{2}(\phi)}{X_{1}(\phi)}
$$

This formula we denominate as the key formula and use it in order to introduce hyperbolic trigonometry on Euclidean plane. Notice that the argument of the exponential function is proportional to the difference between the numerator and the denominator, $X_{1}-X_{2}=x_{1}-x_{2}$, and this difference does not depend on the parameter $\phi$.

\subsection{Elements of Right-Angled Triangle as Functions of a Hyper-} bolic Trigonometry. Let $\triangle A B C$ be a right-angled triangle with right angle at $C$. Denote the sides by $a, b$, the hypotenuse by $c$, and the angles opposite to $a, b, c$ by $A, B, C$, correspondingly. Traditionally interrelations between angles and sides of a triangle are described by the trigonometry via periodical sine-cosine functions. The periodical functions of the circular angles are defined via the ratios

$$
\sin B=\frac{b}{c}, \quad \tan B=\frac{b}{a} .
$$

Notice that these two ratios are functions of the same angle $B$. On making use of formula (9) we are able to introduce the hyperbolic trigonometry besides the circular trigonometry. Define the following relationships:

$$
\frac{c+a}{c-a}=\exp (2 \xi)
$$

From this equation it follows that

$$
\frac{a}{c}=\tanh (\xi), \quad \frac{a}{b}=\sinh (\xi) .
$$

In this way we arrive to the following interrelations between circular and hyperbolic functions:

$$
\cos B=\frac{a}{c}=\tanh (\xi), \quad \tan B=\frac{b}{a}=\frac{1}{\sinh (\xi)} .
$$

Now let us introduce the following geometrical motion; namely, change position of the point $A$ along the line $A C$ (see, Figure 1). Then, the sides $b$ and $c$ will change while the side $a$ will not change. Since the length $a$ is a constant of this evolution, in agreement with key formula (9) we rewrite (11) as follows:

$$
\frac{c+a}{c-a}=\exp (2 a \phi)
$$

that is, the argument of exponential function is proportional to $a: \xi=a \phi$, where $\phi$ is a parameter of the evolution. Formulae in (13) are rewritten as

$$
\cos B=\frac{a}{c}=\tanh (a \phi), \quad \tan B=\frac{b}{a}=\frac{1}{\sinh (a \phi)} .
$$

From these formulas it is derived that

$$
\cot B=\sinh (a \phi)
$$

or,

$$
\tan \frac{B}{2}=\exp (-a \phi)
$$




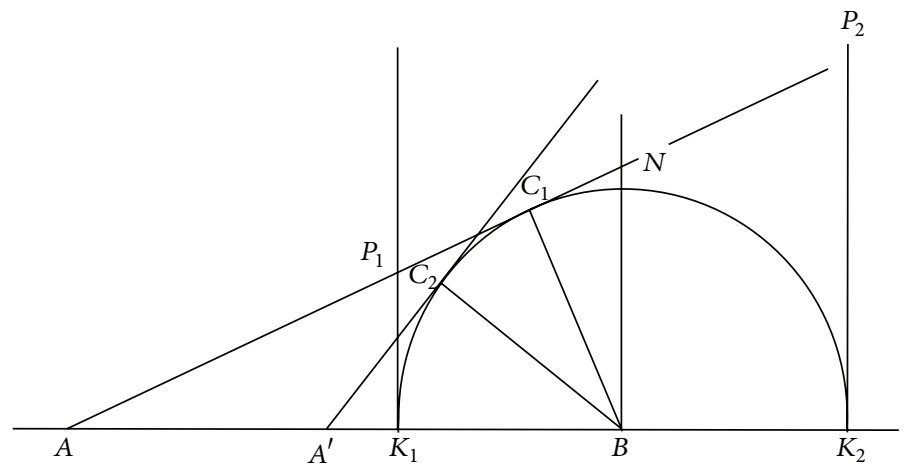

Figure 2: Semicircle and lines tangent to the semicircle.

Install the triangle $\triangle A B C$ in such a way that the side $b=$ $A C$ lies on the line $L$, and the side $a=B C$ is perpendicular to this line at the point $C$

In Figure 1 the line $A B$ moves in such a way that cuts line $L$ at points $A, A^{\prime}, A^{\prime \prime}$, and so forth. Now, let us recall the problem of parallel lines in geometry [7]. Let the ray $A B$ tend to a definite limiting position; in Figure 1 this position is given by line $L_{1}$. As the point $A$ moves along line $L$ away from point $C$ there are two possibilities to consider.

(1) In Euclidean geometry, the angle between lines $L_{1}$ and $B C$ is equal to right angle.

(2) The hypothesis of hyperbolic geometry is that this angle is less than the right angle.

The most fundamental formula of the hyperbolic geometry is the formula connecting the angle of parallelism $\Pi(a)$ and the length $a$ of the perpendicular from the given point to the given line. In order to establish those relationships the concept of horocycles, some circles with center and axis at infinity, was introduced [8]. The great theorem which enables one to introduce the circular functions, sines, and cosines of an angle is that the geometry of shortest lines (horocycles) traced on horosphere is the same as plane Euclidean geometry. The function connecting the angle of parallelism with the distance $a$ is given by

$$
\exp \left(-\frac{a}{\kappa}\right)=\tan \frac{\Pi(a)}{2} .
$$

Now, we introduce the value inverse to $\phi$ by $\mathbf{K}=1 / \phi$ and rewrite (17) in the form

$$
\exp \left(-\frac{a}{\mathbf{K}}\right)=\tan \frac{B}{2}
$$

Let the point $A$ run away from the point $C$ to infinity. The following two cases can be considered.

(1) $\phi$ tends to zero, and $\mathbf{K}$ tends to infinity; then the angle $B$ will tend to right angle.

This is true in Euclidean plane.

(2) Suppose that $\phi$, and $\mathbf{K}$ and the angle $B$ go to some limited values,

$$
\lim _{A C \longrightarrow \infty} \mathbf{K}=\kappa, \quad \lim B=\Pi(a) .
$$

In this way, we have established connection of formula (17) with the main formula of hyperbolic geometry (19).

2.3. Rotational Motion of a Line Tangent to the Semicircle. The concept of the circular angle in Euclidean plane is intimately related to the figure of a circle and to motion of a point along the circumference. The hyperbolic angle is also related to the circle because of a motion along the circumference coherence with the motion along the hyperbola [9].

Consider semicircle $\mathbf{C}$ (Figure 2) with end-points and the center on $x$-axis. Denote by $B$ the center and by $K_{1}, K_{2}$ the end-points of the semicircle. Through end-points of the semicircle, $K_{1}, K_{2}$ erect the lines parallel to vertical axis, $Y$ axis. Draw a line tangent to the semicircle at the point $C$; this line crosses $x$-axis at the point $A$ and intersects with the vertical lines at points $P_{1}$ and $P_{2}$. Draw a line parallel to $Y$-axis from the center $B$ which crosses $C$ at the top point $N$.

Denote by $r$ radius of the circle, so that $r=B N=B C$ and $r=K_{1} K_{2}$. Denote by $B$ the angle $\angle A B C$. The triangle $\triangle A B C$ is a right triangle, so that

$$
(A B)^{2}-(A C)^{2}=r^{2} .
$$

Consider rotational motion of the line tangent to the semicircle at the point $\mathbf{C}$. In Figure 2, two positions of this line are given by lines $A^{\prime} C_{2}$ and $A C_{1}$. When the point Cruns from end point $K_{1}$ to the top-point $N$, the point $A$ runs along $x$ axis from point $K_{1}$ to infinity. During this motion the triangle formed by the line tangent to the semicircle, the $x$-axis and the radius of the circle remains to be right angled. This is exactly the case considered in Section 2.2, consequently, we can apply formula (13). According to (13) we write

$$
A B=r \operatorname{coth}(r \phi)=\frac{r}{\cos B}, \quad A C_{1}=\frac{r}{\sinh (r \phi)}=r \tan B .
$$

Since $\triangle A B C_{1} \sim \triangle A K_{1} P_{1}$, we have

$$
\begin{gathered}
\frac{P_{1} K_{1}}{A K_{1}}=\frac{r}{A C_{1}}=\sinh (r \phi), \\
P_{1} K_{1}=A K_{1} \sinh (r \phi)=(A B-r) \sinh (r \phi) \\
=r \exp (-r \phi), \quad P_{2} K_{2}=r \exp (r \phi) .
\end{gathered}
$$


On the basis of obtained formulae the following relationships between circular and hyperbolic trigonometric functions are established:

$$
\begin{gathered}
\sin (B)=\frac{1}{\cosh (r \phi)}, \quad \cot (B)=\sinh (r \phi), \\
\cos (B)=\tanh (r \phi) .
\end{gathered}
$$

If we make the point $C$ tend to the top of semicircle $N$, the hyperbolic angle $\phi$ will tend to zero. When the point $C$ tends to end-point $K_{1}$, the hyperbolic angle tends to infinity. Thus, the hyperbolic angle is measured from the point $N$, the top of the semicircle. Consider two different positions of the tangent line, corresponding to two positions $C_{1}, C_{2}$, with hyperbolic angles $\phi_{2}$ and $\phi_{1}$. According to key formula (6) we write

$$
\begin{aligned}
& \exp \left(\left(x_{2}-x_{1}\right) \phi_{2}\right)=\frac{x_{2}-D_{2}}{x_{1}-D_{2}} \\
& \exp \left(\left(x_{2}-x_{1}\right) \phi_{1}\right)=\frac{x_{2}-D_{1}}{x_{1}-D_{1}}
\end{aligned}
$$

The hyperbolic angle $\phi_{2}$ corresponds to the arc $\smile N C_{2}$, and the hyperbolic angle $\phi_{1}$ corresponds to the arc $\smile N C_{1}$. Then we suppose that the difference in the hyperbolic angles $\phi_{2}-\phi_{1}$ will correspond to the arc $-C_{2} C_{1}$. From (26) it follows that

$$
\begin{gathered}
\exp \left(x_{2}-x_{1}\right)\left(\phi_{2}-\phi_{1}\right)=\frac{x_{2}-D_{2}}{x_{1}-D_{2}} \frac{x_{1}-D_{1}}{x_{2}-D_{1}}, \\
D_{k}=D\left(\phi_{k}\right), \quad k=1,2 .
\end{gathered}
$$

It is seen, in the right-hand side we have the cross-ratio. Now, let recall definition of the distance between two points of the geodesic line in the Poincaré model. Let $z, w \in H$, and let $z_{1}$ and $w_{1}$ be end points of a geodesic line passing through $z$ and $w$. Then the distance between these points is defined by formula

$$
\rho\left(z_{1}, z_{3}\right)=\log \left(z_{1}, z_{2} ; z_{3}, z_{4}\right)
$$

where

$$
\left(z_{1}, z_{2} ; z_{3}, z_{4}\right):=\frac{z_{2}-z_{1}}{z_{4}-z_{1}} \frac{z_{4}-z_{3}}{z_{2}-z_{3}}
$$

is the cross-ratio. Comparing (29) with (27) we come to the following correspondence: $z_{2}=x_{2}, z_{4}=x_{1}, z_{1}=D_{2}$, $z_{3}=D_{1}$. Notice, however, in our construction $D_{k}, k=1,2$ are projections of $z_{1}, z_{3}$ on $X$-axis. The semicircle $\mathbf{C}$ is the geodesic line, and $x_{1}, x_{2}$ are end-points of the geodesic line.

\section{Relationships between Elements of Three Intersecting Semicircles}

3.1. Hyperbolic Cosine-Sine Functions of Arcs of the Semicircle. The key formula (6) admits to define hyperbolic trigonometric functions of the arcs originated from the top $N$ of the semicircle C. In order to determine trigonometric functions of the arcs with arbitrary end-points on the semicircle we have to use formula (27). Consider arc $\smile C_{1} C_{2}$ is defined in the first quadrant of the semicircle with end-points at $C_{1}$ and $C_{2}$. The arc $\smile C_{1} C_{2}$ can be presented as difference of two arcs, both originated from the top of the semicircle:

$$
\smile C_{1} C_{2}=\smile N C_{1}-\smile N C_{2} .
$$

Denote by $a_{1}, a_{2}$ the angles formed by radiuses $B C_{1}$ and $B C_{2}$ with $x$-axis, correspondingly, where $B$ is a center of the circle. Denote the hyperbolic angle corresponding to the arcs $\smile N C_{k}, k=1,2$ by $\xi\left(N C_{k}\right), k=1,2$. Then the functions

$$
\begin{array}{cc}
\cosh \xi\left(N C_{1}\right), & \cosh \xi\left(N C_{2}\right), \\
\sinh \xi\left(N C_{1}\right), & \sinh \xi\left(N C_{2}\right)
\end{array}
$$

are expressed via circular trigonometric functions according to formulae (25):

$$
\begin{array}{ll}
\cosh \xi\left(N C_{1}\right)=\frac{1}{\sin a_{1}}, & \cosh \xi\left(N C_{2}\right)=\frac{1}{\sin a_{2}} \\
\sinh \xi\left(N C_{1}\right)=\cot a_{1}, & \sinh \xi\left(N C_{2}\right)=\cot a_{2} .
\end{array}
$$

Then, by taking into account additional formulae

$$
\begin{aligned}
\cosh \xi\left(C_{1} C_{2}\right)= & \cosh \xi\left(N C_{1}\right) \cosh \xi\left(N C_{2}\right) \\
& -\sinh \xi\left(N C_{1}\right) \sinh \xi\left(N C_{2}\right), \\
\sinh \xi\left(C_{1} C_{2}\right)= & \sinh \xi\left(N C_{1}\right) \cosh \xi\left(N C_{2}\right) \\
& -\cosh \xi\left(N C_{1}\right) \sinh \xi\left(N C_{2}\right),
\end{aligned}
$$

we get hyperbolic trigonometric functions of the arcs with arbitrary end-points on the semicircle expressed via periodic trigonometric functions:

$$
\begin{aligned}
& \cosh \xi\left(C_{1} C_{2}\right)=\frac{1-\cos a_{1} \cos a_{2}}{\sin a_{1} \sin a_{2}}, \\
& \sinh \xi\left(C_{1} C_{2}\right)=\frac{\cos a_{1}-\cos a_{2}}{\sin a_{1} \sin a_{2}} .
\end{aligned}
$$

Compare with analogous formula from Poincaré model ([3], formula (1.2.6)) given by

$$
\cosh \rho(z, w)=1+\frac{|z-w|^{2}}{2 \operatorname{Im}(z) \operatorname{Im}(w)} .
$$

Two complex numbers

$$
z=v_{0}+i v, \quad w=w_{0}+i w
$$

are related to the radius of the semicircle and the angles $a_{1}, a_{2}$ by

$$
\begin{array}{ll}
v=r \sin a_{2}, & v_{0}=r \cos a_{2}, \\
w=r \sin a_{1}, & w_{0}=r \sin a_{1} .
\end{array}
$$




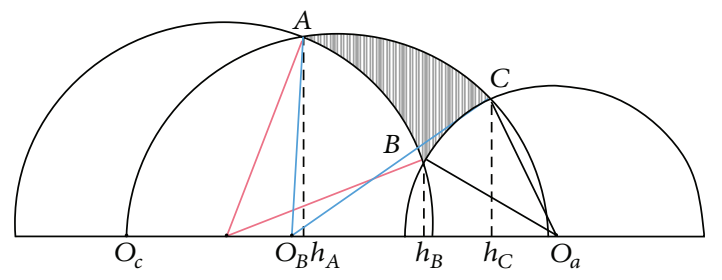

Figure 3: Triangle formed by arcs of intersecting semicircles.

Then,

$$
\begin{aligned}
\cosh \xi\left(C_{1} C_{2}\right) & =\frac{1-\cos a_{1} \cos a_{2}}{\sin a_{1} \sin a_{2}}=\frac{r^{2}-v_{0} w_{0}}{w v} \\
& =1+\frac{\left(v_{0}-w_{0}\right)^{2}+(v-w)^{2}}{2 w v}=\cosh \rho(z, w) .
\end{aligned}
$$

\subsection{Relationships between Elements of the Triangle Bounded} by Arcs of the Intersecting Semicircles. In Figure 3 three intersecting semicircles with centers installed on horizontal axis at the points $O_{a}, O_{b}$, and $O_{c}$ are presented. Intersections of the semicircles form triangle $\triangle A B C$ bounded by the arcs $B C, \smile A B, \smile A C$. For each arc we can put in correspondence the hyperbolic angle. Denote the hyperbolic angles by $a, b, c$, where $a, b, c$ consecutively will correspond to the arcs $B C, \smile A C, \smile A B$.

Connect vertices of the triangle with centers of the circle by corresponding radiuses. Denote by $a_{k}, b_{k}, c_{k}, k=1,2$ the angles bounded by the radiuses and $x$-axis, where $a_{1}>a_{2}>0$, $b_{1}>b_{2}>0, c_{1}>c_{2}>0$. By making use of (34a) define hyperbolic cosine-sine functions corresponding to bounding segments:

$$
\begin{array}{ll}
\cosh a=\frac{1-\cos a_{1} \cos a_{2}}{\sin a_{1} \sin a_{2}}, & \sinh a=\frac{\cos a_{1}-\cos a_{2}}{\sin a_{1} \sin a_{2}}, \\
\cosh b=\frac{1-\cos b_{1} \cos b_{2}}{\sin b_{1} \sin b_{2}}, & \sinh b=\frac{\cos b_{1}-\cos b_{2}}{\sin b_{1} \sin b_{2}}, \\
\cosh c=\frac{1-\cos c_{1} \cos c_{2}}{\sin c_{1} \sin c_{2}}, & \sinh c=\frac{\cos c_{1}-\cos c_{2}}{\sin c_{1} \sin c_{2}} .
\end{array}
$$

The usual notion of the angle is used that is, the angle between two curves is defined as an angle between their tangent lines. Let the angles $\alpha, \beta, \delta$ be angles at the verteices $A, B, C$, correspondingly. For these angles we can define thier proper cosine and sine functions. The angles of the triangle $\widetilde{\triangle A B C} \alpha, \beta, \delta$ are closely related to angles $a_{1}, a_{2}, b_{1}, b_{2}, c_{1}, c_{2}$. From Figure 3 we find the following relationships between them:

$$
\beta=a_{2}+c_{2}, \quad \delta=\pi-a_{1}-b_{2}, \quad \alpha=b_{1}-c_{1} .
$$

Then,

$$
\begin{aligned}
& \cos \alpha=\cos b_{1} \cos c_{1}+\sin b_{1} \sin c_{1}, \\
& \cos \beta=\cos a_{2} \cos c_{2}-\sin a_{2} \sin c_{2}, \\
& \cos \delta=-\cos b_{2} \cos a_{1}+\sin b_{2} \sin a_{1}, \\
& \sin \alpha=\sin b_{1} \cos c_{1}-\cos b_{1} \sin c_{1}, \\
& \sin \beta=\sin a_{2} \cos c_{2}+\cos a_{2} \sin c_{2}, \\
& \sin \delta=\sin b_{2} \cos a_{1}+\cos b_{2} \sin a_{1} .
\end{aligned}
$$

Denote distances between centers by

$$
O_{c b}=O_{c} O_{b}, \quad O_{b a}=O_{b} O_{a}, \quad O_{a c}=O_{a} O_{c} .
$$

The theorem of sines employed for triangles $\mathrm{O}_{c} A \mathrm{O}_{b}, \mathrm{O}_{b} \mathrm{CO}_{a}$, $\mathrm{O}_{c} B \mathrm{O}_{a}$ gives six relations of type

$$
\begin{aligned}
& \frac{\sin \alpha}{O_{c b}}=\frac{\sin c_{1}}{r_{b}}=\frac{\sin b_{1}}{r_{c}}, \\
& \frac{\sin \delta}{O_{b a}}=\frac{\sin a_{1}}{r_{b}}=\frac{\sin b_{2}}{r_{a}}, \\
& \frac{\sin \beta}{O_{a c}}=\frac{\sin c_{2}}{r_{a}}=\frac{\sin a_{2}}{r_{c}} .
\end{aligned}
$$

From these relations the first set of main relationships follows.

Relation 1. Consider

$$
\begin{gathered}
r_{a} \sin a_{1}=r_{b} \sin b_{2}, \quad r_{c} \sin c_{2}=r_{a} \sin a_{2}, \\
r_{c} \sin c_{1}=r_{b} \sin b_{1} .
\end{gathered}
$$

From the draught in Figure 3 it is seen that

$$
O_{a c}=O_{b a}+O_{c b}
$$

where

$$
O_{a c}=r_{c} \cos c_{2}+r_{a} \cos a_{2}, \quad O_{b a}=r_{a} \cos a_{1}+r_{b} \cos b_{2} .
$$

Hence,

$$
O_{c b}=r_{c} \cos c_{2}+r_{a} \cos a_{2}-r_{a} \cos a_{1}-r_{b} \cos b_{2} .
$$

From vertices of $\widetilde{\triangle} A B C$ erect lines perpendicular to horizontal line intersect with $x$-axis at points $h_{A}, h_{B}, h_{C}$, correspondingly. From Figure 3 we find that

$$
O_{c b}=O_{c} h_{A}-h_{A} O_{b}=r_{c} \cos c_{1}-r_{b} \cos b_{1} .
$$

By equating (47) with (48) we arrive to another main relationship between radii and angles. 
Relation 2. One has

$$
\begin{aligned}
r_{c} \cos c_{1}-r_{b} \cos b_{1}= & r_{c} \cos c_{2}+r_{a} \cos a_{2}-r_{a} \cos a_{1} \\
& -r_{b} \cos b_{2} .
\end{aligned}
$$

We will effect a simplification by using the following designations:

$$
\begin{array}{ll}
r_{a} \cos a_{1}=w_{01}, & r_{a} \sin a_{1}=w_{1}, \\
r_{a} \cos a_{2}=v_{01}, & r_{a} \sin a_{2}=v_{1}, \\
r_{b} \cos b_{1}=w_{02}, & r_{b} \sin b_{1}=w_{2}, \\
r_{b} \cos b_{2}=v_{02}, & r_{b} \sin b_{2}=v_{2}, \\
r_{c} \cos c_{1}=w_{03}, & r_{c} \sin c_{1}=w_{3}, \\
r_{c} \cos c_{2}=v_{03}, & r_{c} \sin c_{2}=v_{3} .
\end{array}
$$

In these designations formulae (40a), (40b), (40c) and (41a), (41b), (41c) are written as follows:

$$
\begin{aligned}
& r_{b} r_{c} \sin \alpha=w_{2} w_{03}-w_{02} w_{3}, \\
& r_{b} r_{c} \cos \alpha=w_{02} w_{03}+w_{2} w_{3}, \\
& r_{a} r_{c} \sin \beta=v_{1} v_{03}+v_{01} v_{3}, \\
& r_{a} r_{c} \cos \beta=v_{01} v_{03}-v_{1} v_{3}, \\
& r_{a} r_{b} \sin \delta=v_{2} w_{01}+v_{02} w_{1}, \\
& r_{a} r_{b} \cos \delta=v_{2} w_{1}-v_{02} w_{01} .
\end{aligned}
$$

Formulae (38a), (38b), (38c) for hyperbolic sines and cosines are rewritten as follows:

$$
\begin{array}{ll}
\cosh a=\frac{r_{a}^{2}-w_{01} v_{01}}{w_{1} v_{1}}, & \sinh a=r_{a} \frac{w_{01}-v_{01}}{w_{1} v_{1}}, \\
\cosh b=\frac{r_{b}^{2}-w_{02} v_{02}}{w_{2} v_{2}}, & \sinh b=r_{b} \frac{w_{02}-v_{02}}{w_{2} v_{2}}, \\
\cosh c=\frac{r_{c}^{2}-w_{03} v_{03}}{w_{3} v_{3}}, & \sinh c=r_{c} \frac{w_{03}-v_{03}}{w_{3} v_{3}} .
\end{array}
$$

In the designations equations of Relation 1 are written as

$$
x:=w_{2}=w_{3}, \quad y:=v_{1}=v_{3}, \quad z:=w_{1}=v_{2} .
$$

Equations (43a), (43b), (43c)-(46) are rewritten as follows:

$$
\begin{gathered}
O_{c b}=w_{03}-w_{02}, \quad O_{a c}=v_{03}+v_{01}, \\
O_{b a}=w_{01}+v_{02} .
\end{gathered}
$$

Correspondingly, Relation 2 takes the form

$$
w_{03}-w_{02}=v_{03}+v_{01}-w_{01}-v_{02} .
$$

This expresses the fact that $O_{c a}$ is a sum of $O_{c b}$ and $O_{b a}$. Notice that (55) can be rewritten also in another equivalent form namely,

$$
w_{03}-v_{03}=w_{02}-v_{02}-\left(w_{01}-v_{01}\right)
$$

Denote the segments projections of sides of $\widetilde{\triangle} A B C$ on $x$-axis by $\mathbf{P}(A C)=h_{A} h_{C}, \mathbf{P}(A B)=h_{A} h_{B}, \mathbf{P}(B C)=h_{B} h_{C}$. From Figure 3 it is seen that

$$
\mathbf{P}(A C)=\mathbf{P}(A B)+\mathbf{P}(B C),
$$

where

$$
\begin{gathered}
\mathbf{P}(B C)=w_{01}-v_{01}, \quad \mathbf{P}(A C)=w_{02}-v_{02}, \\
\mathbf{P}(A B)=w_{03}-v_{03} .
\end{gathered}
$$

\section{Hyperbolic Law of Cosines I for the Triangle Formed by Intersection of Three Semicircles}

The main aim of this section is to prove the hyperbolic law theorem of cosines I for the triangle $\widetilde{\triangle} A B C$ formed by arcs of intersecting semicircles with centers installed on $x$-axis (Figure 3). This law is given by the following set of equations:

$$
\begin{aligned}
& \cosh c=\cosh a \cosh b-\sinh a \sinh b \cos \delta, \\
& \cosh b=\cosh a \cosh c-\sinh c \sinh a \cos \beta, \\
& \cosh a=\cosh c \cosh b-\sinh c \sinh b \cos \alpha .
\end{aligned}
$$

Theorem 3 (theorem of cosines I). The following equation for elements of the triangle $\widetilde{\triangle} A B C$ formed by arcs of three intersecting semicircles holds true

$$
\cosh c=\cosh a \cosh b-\sinh a \sinh b \cos \delta,
$$

where $\cosh c, \cosh a, \cosh b, \sinh a, \sinh b, \cos \delta$ are defined by formulae (51a), (51b), (51c)-(52).

Proof. Square both sides of Relation 2 to obtain

$$
\begin{aligned}
\left(w_{03}-v_{03}\right)^{2}= & \left(w_{02}-v_{02}\right)^{2}+\left(w_{01}-v_{01}\right)^{2} \\
& -2\left(w_{02}-v_{02}\right)\left(w_{01}-v_{01}\right)
\end{aligned}
$$

and evaluate this equality by taking into account formulae (51a), (51b), (51c)-(52). First of all, evaluate the left-hand side of this equation as follows:

$$
\begin{aligned}
\left(w_{03}-v_{03}\right)^{2} & =v_{03}^{2}+w_{03}^{2}-2 v_{03} w_{03} \\
& =v_{03}^{2}+w_{03}^{2}-2 r_{c}^{2}+2\left(r_{c}^{2}-v_{03} w_{03}\right),
\end{aligned}
$$

where

$$
2 r_{c}^{2}=w_{03}^{2}+w_{3}^{2}+v_{03}^{2}+v_{3}^{2} .
$$

Use (63) and write (62) as follows:

$$
\begin{aligned}
v_{03}^{2}+w_{03}^{2}-2 r_{c}^{2} & =v_{03}^{2}+w_{03}^{2}-\left(w_{03}^{2}+w_{3}^{2}+v_{03}^{2}+v_{3}^{2}\right) \\
& =-\left(w_{3}^{2}+v_{3}^{2}\right) .
\end{aligned}
$$

Equation (61) takes the following form:

$$
\begin{aligned}
-\underbrace{\left(w_{3}^{2}+v_{3}^{2}\right)}+2\left(r_{c}^{2}-v_{03} w_{03}\right)= & \left(w_{02}-v_{02}\right)^{2}+\left(w_{01}-v_{01}\right)^{2} \\
& -2\left(w_{02}-v_{02}\right)\left(w_{01}-v_{01}\right) .
\end{aligned}
$$


The underlined term passes to the right-hand side of the equation. Then in the left-hand side remains the expression

$$
2\left(r_{c}^{2}-v_{03} w_{03}\right)=2 v_{3} w_{3} \cosh c .
$$

Dividing both sides of the obtained equation by $v_{3} w_{3}$, we get

$$
\begin{gathered}
2 \cosh c=\frac{1}{v_{3} w_{3}}\left(w_{3}^{2}+v_{3}^{2}+\left(v_{02}-w_{02}\right)^{2}+\left(v_{01}-w_{01}\right)^{2}\right. \\
\left.-2\left(v_{02}-w_{02}\right)\left(v_{01}-w_{01}\right)\right)
\end{gathered}
$$

according to Relation $1 w_{3}=w_{2}, v_{3}=v_{1}$. These relations make true the following equation

$$
\frac{1}{w_{3} v_{3}}=\frac{1}{w_{2} v_{2}} \frac{1}{w_{1} v_{1}} v_{2} w_{1} .
$$

On making use this formula in the right-hand side of (67) we come to the following equation:

$$
\begin{aligned}
2 \cosh c= & \frac{1}{w_{2} v_{2}} \frac{1}{w_{1} v_{1}} \\
& \times\left\{v_{2} w_{1}\left(w_{3}^{2}+v_{3}^{2}+\left(v_{02}-w_{02}\right)^{2}+\left(v_{01}-w_{01}\right)^{2}\right)\right\} \\
& -\underbrace{2 \frac{1}{w_{2} v_{2}} \frac{1}{w_{1} v_{1}} v_{2} w_{1}\left(v_{02}-w_{02}\right)\left(v_{01}-w_{01}\right) .}
\end{aligned}
$$

Evaluate now the underlined term, which we firstly write as follows:

$$
\begin{aligned}
2\left(v_{02}\right. & \left.-w_{02}\right)\left(v_{01}-w_{01}\right) \frac{1}{w_{2} v_{2}} \frac{1}{w_{1} v_{1}} v_{2} w_{1} \\
& =2 \frac{v_{2} w_{1}}{r_{a} r_{b}} \frac{r_{b}\left(v_{02}-w_{02}\right)}{w_{2} v_{2}} \frac{r_{a}\left(v_{01}-w_{01}\right)}{w_{1} v_{1}} .
\end{aligned}
$$

From the second equation of (51c) we have

$$
\frac{v_{2} w_{1}}{r_{a} r_{b}}=\cos \delta+\frac{v_{02} w_{01}}{r_{a} r_{b}} .
$$

By making use of (71) in (70), evaluate (70) as follows:

$$
\begin{aligned}
2 \frac{v_{2} w_{1}}{r_{a} r_{b}} \frac{r_{b}\left(v_{02}-w_{02}\right)}{w_{2} v_{2}} \frac{r_{a}\left(v_{01}-w_{01}\right)}{w_{1} v_{1}} & \\
= & 2\left(\cos \delta+\frac{v_{02} w_{01}}{r_{a} r_{b}}\right) \frac{r_{b}\left(v_{02}-w_{02}\right)}{w_{2} v_{2}} \frac{r_{a}\left(v_{01}-w_{01}\right)}{w_{1} v_{1}} \\
= & 2 \cos \delta \frac{r_{b}\left(v_{02}-w_{02}\right)}{w_{2} v_{2}} \frac{r_{a}\left(v_{01}-w_{01}\right)}{w_{1} v_{1}} \\
& -2 v_{02} w_{01} \frac{\left(v_{02}-w_{02}\right)}{w_{2} v_{2}} \frac{\left(v_{01}-w_{01}\right)}{w_{1} v_{1}} \\
= & 2 \cos \delta \sinh a \sinh b-2 v_{02} w_{01} \frac{\left(v_{02}-w_{02}\right)}{w_{2} v_{2}} \frac{\left(v_{01}-w_{01}\right)}{w_{1} v_{1}} .
\end{aligned}
$$

Replace the underlined term of (69) by (72), and pass expression containing sines and cosines to the left-hand side of the obtained equation. As a result, we come to the following equation:

$2 \cosh c-2 \cos \delta \sinh b \sinh a$

$$
\begin{gathered}
=\frac{1}{w_{2} v_{2} w_{1} v_{1}}\left\{v _ { 2 } w _ { 1 } \left(w_{2}^{2}+v_{1}^{2}+\left(w_{02}-v_{02}\right)^{2}\right.\right. \\
\left.+\left(w_{01}-v_{01}\right)^{2}\right)-2 v_{02} w_{01} \\
\left.\times\left(w_{02}-v_{02}\right)\left(w_{01}-v_{01}\right)\right\}
\end{gathered}
$$

By applying the elementary algebra one may show that (see, [10])

$$
\begin{aligned}
v_{2} w_{1}\left(w_{2}^{2}+v_{1}^{2}+\left(w_{02}-v_{02}\right)^{2}+\left(w_{01}-v_{01}\right)^{2}\right) \\
-2 v_{02} w_{01}\left(w_{02}-v_{02}\right)\left(w_{01}-v_{01}\right) \\
=2\left(r_{a}^{2}-v_{01} w_{01}\right)\left(r_{b}^{2}-v_{02} w_{02}\right) .
\end{aligned}
$$

Thus, in the right-hand side of (73) we have

$$
2 \frac{1}{w_{1} v_{1} v_{2} w_{2}}\left(r_{a}^{2}-v_{01} w_{01}\right)\left(r_{b}^{2}-v_{02} w_{02}\right),
$$

which according to formulae (52) is nothing else than

$$
2 \cosh a \cosh b \text {. }
$$

By using it in (73) we arrive to the following equation:

$$
2 \cosh c-2 \cosh a \cosh b=2 \cos \delta \sinh a \sinh b .
$$

\section{Hyperbolic Laws of Sines and Cosines II}

The main task of this section is to prove hyperbolic law (theorem) of sines, which is given by the formulae

$$
\frac{\sinh a}{\sin \alpha}=\frac{\sinh b}{\sin \beta}=\frac{\sinh c}{\sin \delta},
$$

and the hyperbolic law (theorem) of cosines II given by the formulae

$$
\begin{aligned}
& \cos \delta=-\cos \alpha \cos \beta-\sin \alpha \sin \beta \cosh c, \\
& \cos \beta=-\cos \alpha \cos \delta-\sin \alpha \sin \delta \cosh b \\
& \cos \alpha=-\cos \beta \cos \delta-\sin \delta \sin \beta \cosh a
\end{aligned}
$$

\subsection{Hyperbolic Theorem of Sines and Its Geometrical Interpre- tation on Euclidean Plane}

Theorem 4. The ratios of projections of the sides of triangle $\widetilde{\triangle} A B C$ on $x$-axis to corresponding distances between centers of the semicircles are equal to each other. 
Proof. Projections of the sides of $\widetilde{\triangle} A B C$ are given by formulae

$$
\begin{gathered}
\mathbf{P}_{b c}=r_{a} \cos a_{1}-r_{a} \cos a_{2}, \quad \mathbf{P}_{c a}=r_{b} \cos b_{1}-r_{b} \cos b_{2}, \\
\mathbf{P}_{a b}=r_{c} \cos c_{1}-r_{c} \cos c_{2} .
\end{gathered}
$$

Distances between centers of the circles have been defined as (see, (46) and (47))

$$
\begin{gathered}
O_{c a}=r_{c} \cos c_{2}+r_{a} \cos a_{2}, \quad O_{b a}=r_{a} \cos a_{1}+r_{b} \cos b_{2}, \\
O_{c b}=r_{c} \cos c_{1}-r_{b} \cos b_{1}, \\
\mathbf{P}_{c a}=\mathbf{P}_{b c}+\mathbf{P}_{a b}, \quad O_{c a}=O_{b c}+O_{a b} .
\end{gathered}
$$

Relation 1 given by the set of equations

$$
\begin{gathered}
r_{a} \sin a_{1}=r_{b} \sin b_{2}, \quad r_{c} \sin c_{2}=r_{a} \sin a_{2}, \\
r_{c} \sin c_{1}=r_{b} \sin b_{1}
\end{gathered}
$$

raises to the second power,

$$
\begin{aligned}
& r_{a}^{2}-r_{a}^{2} \cos ^{2} a_{1}=r_{b}^{2}-r_{b}^{2} \cos ^{2} b_{2}, \\
& r_{c}^{2}-r_{c}^{2} \cos ^{2} c_{2}=r_{a}^{2}-r_{a}^{2} \cos ^{2} a_{2}, \\
& r_{c}^{2}-r_{c}^{2} \cos ^{2} c_{1}=r_{b}^{2}-r_{b}^{2} \cos ^{2} b_{1} .
\end{aligned}
$$

Then, square distances $O_{i k}^{2}, i, k=a, b, c$ and use (86). We get

$$
\begin{aligned}
& O_{c a}^{2}=r_{c}^{2} \cos ^{2} c_{2}+r_{a}^{2} \cos ^{2} a_{2}+2 r_{c} r_{a} \cos c_{2} \cos a_{2}, \\
& O_{b a}^{2}=r_{a}^{2} \cos ^{2} a_{1}+r_{b}^{2} \cos ^{2} b_{2}+2 r_{a} r_{b} \cos a_{1} \cos b_{2}, \\
& O_{c b}^{2}=r_{c}^{2} \cos ^{2} c_{1}+r_{b}^{2} \cos ^{2} b_{1}-2 r_{c} r_{b} \cos c_{1} \cos b_{1} .
\end{aligned}
$$

Combine (86) with (87); in this way we come to the following system of equations:

$$
\begin{aligned}
& \text { (a) } O_{c a}^{2}=r_{c}^{2}-r_{a}^{2}+2 r_{a} \cos a_{2} O_{a c}, \\
& \text { (b) } O_{c a}^{2}=r_{a}^{2}-r_{c}^{2}+2 r_{c} \cos c_{2} O_{a c}, \\
& \text { (a) } O_{b a}^{2}=r_{a}^{2}-r_{b}^{2}+2 r_{b} \cos b_{2} O_{b a}, \\
& \text { (b) } O_{b a}^{2}=r_{b}^{2}-r_{a}^{2}+2 r_{a} \cos a_{1} O_{b a}, \\
& \text { (a) } O_{c b}^{2}=r_{b}^{2}-r_{c}^{2}+2 r_{c} \cos c_{1} O_{c b}, \\
& \text { (b) } O_{c b}^{2}=r_{c}^{2}-r_{b}^{2}-2 r_{b} \cos b_{1} O_{c b} \text {. }
\end{aligned}
$$

From these equations the cosines of the angles $a_{1}, a_{2}, b_{1}, b_{2}$, $c_{1}, c_{2}$ are expressed:

$$
\begin{array}{ll}
\frac{O_{b a}^{2}-r_{b}^{2}+r_{a}^{2}}{2 r_{a} O_{b a}}=\cos a_{1}, & \frac{O_{c a}^{2}-r_{c}^{2}+r_{a}^{2}}{2 r_{a} O_{a c}}=\cos a_{2}, \\
\frac{O_{c b}^{2}-r_{b}^{2}+r_{c}^{2}}{2 r_{c} O_{c b}}=\cos c_{1}, & \frac{O_{c a}^{2}-r_{a}^{2}+r_{c}^{2}}{2 r_{c} O_{a c}}=\cos c_{2}, \\
\frac{-O_{c b}^{2}+r_{c}^{2}-r_{b}^{2}}{2 r_{b} O_{c b}}=\cos b_{1}, & \frac{O_{b a}^{2}-r_{a}^{2}+r_{b}^{2}}{2 r_{b} O_{b a}}=\cos b_{2} .
\end{array}
$$

Having these formulae we may present the projection $\mathbf{P}_{c a}$ as follows

$$
\begin{aligned}
\mathbf{P}_{a c} & =r_{b} \cos b_{1}-r_{b} \cos b_{2} \\
& =\frac{-O_{c b}^{2}+r_{c}^{2}-r_{b}^{2}}{2 O_{c b}}-\frac{O_{b a}^{2}-r_{a}^{2}+r_{b}^{2}}{2 O_{b a}} \\
& =\frac{-O_{c b}^{2} O_{b a}+r_{c}^{2} O_{b a}-r_{b}^{2} O_{b a}-O_{b a}^{2} O_{c b}+r_{a}^{2} O_{c b}-r_{b}^{2} O_{c b}}{2 O_{c b} O_{b a}}
\end{aligned}
$$

The first ratio is presented as follows:

$$
\begin{aligned}
\frac{\mathbf{P}_{c a}}{O_{c a}} & =\frac{-O_{c b} O_{b a}\left(O_{c b}+O_{b a}\right)+r_{c}^{2} O_{b a}-r_{b}^{2}\left(O_{b a}-O_{c b}\right)+r_{a}^{2} O_{c b}}{2 O_{c b} O_{b a} O_{c a}} \\
& =\frac{-O_{c b} O_{b a}\left(O_{c a}\right)+r_{c}^{2} O_{b a}-r_{b}^{2}\left(O_{c a}\right)+r_{a}^{2} O_{c b}}{2 O_{c b} O_{b a} O_{c a}} .
\end{aligned}
$$

Now, in the same way let us calculate the next ratio, namely, $\mathbf{P}_{b c} / \mathrm{O}_{b c}$. Formula for the projection is evaluated as follows:

$$
\begin{aligned}
\mathbf{P}_{b c} & =r_{a} \cos a_{1}-r_{a} \cos a_{2} \\
& =\frac{O_{b a}^{2}-r_{b}^{2}+r_{a}^{2}}{2 O_{b a}}-\frac{O_{c a}^{2}-r_{c}^{2}+r_{a}^{2}}{2 O_{a c}} \\
& =\frac{\left(\left(O_{b a} O_{a c}\left(O_{b a}-O_{c a}\right)-r_{b}^{2} O_{a c}+r_{a}^{2} O_{a c}\right)+r_{c}^{2} O_{b a}-r_{a}^{2} O_{b a}\right)}{2 O_{a c} O_{b a}} .
\end{aligned}
$$

By taking into account equation $-O_{c b}=O_{b a}-O_{a c}$, we get

$$
\mathbf{P}_{b c}=\frac{\left(O_{b a} O_{a c} O_{c b}-r_{b}^{2} O_{a c}+r_{a}^{2} O_{c b}+r_{c}^{2} O_{b a}\right)}{2 O_{a c} O_{b a}} .
$$

Next, let us calculate the ratio

$$
\frac{\mathbf{P}_{b c}}{O_{b c}}=\frac{\left(O_{b a} O_{a c}\left(O_{c b}\right)-r_{b}^{2} O_{a c}+r_{a}^{2}\left(O_{c b}\right)+r_{c}^{2} O_{b a}\right)}{2 O_{a c} O_{b a} O_{b c}} .
$$

This expression coincides with (91); hence,

$$
\frac{\mathbf{P}_{b c}}{O_{b c}}=\frac{\mathbf{P}_{a c}}{O_{a c}} .
$$

By taking into account (84), we arrive to desired relations

$$
\frac{\mathbf{P}_{b c}}{O_{b c}}=\frac{\mathbf{P}_{a c}}{O_{a c}}=\frac{\mathbf{P}_{a b}}{O_{a b}} .
$$

In the sequel come back to designations introduced in Section 3. In these designations (96) is written as follows:

$$
\frac{w_{01}-v_{01}}{w_{03}-w_{02}}=\frac{w_{02}-v_{02}}{v_{03}+v_{01}}=\frac{w_{03}-v_{03}}{w_{01}+v_{02}} .
$$

Theorem 5. The sides and the angles of triangle $\widetilde{\triangle} A B C$ satisfy (78). 
Proof. By using formulas (38a), (38b), and (38c) for $\sinh a$, $\sinh b, \sinh c$ and formulas (41a), (41b), and (41c) for $\sin \alpha$, $\sin \beta, \sin \delta$, we write:

$$
\begin{gathered}
\frac{\sinh a}{\sin \alpha}=\frac{r_{a}\left(w_{01}-v_{01}\right)}{y z}: \frac{x\left(w_{03}-w_{02}\right)}{r_{b} r_{c}} \\
=\frac{w_{01}-v_{01}}{w_{03}-w_{02}} \frac{x y z}{r_{a} r_{b} r_{c}}, \\
\frac{\sinh b}{\sin \beta}=\frac{w_{02}-v_{02}}{r_{b} x z}: \frac{y\left(v_{03}+v_{01}\right)}{r_{a} r_{c}}=\frac{w_{02}-v_{02}}{v_{03}+v_{01}} \frac{x y z}{r_{a} r_{b} r_{c}}, \\
\frac{\sinh c}{\sin \delta}=\frac{w_{03}-v_{03}}{r_{c} y x}: \frac{z\left(w_{01}+v_{02}\right)}{r_{b} r_{a}}=\frac{w_{03}-v_{03}}{w_{01}+v_{02}} \frac{x y z}{r_{a} r_{b} r_{c}} .
\end{gathered}
$$

It is seen that these equations contain a common factor which is symmetric with respect to $a, b, c$ and $x, y, z$. Multiply all equations of (97) by this factor. We arrive to (78).

Theorem 6. The sides and the angles of triangle $\widetilde{\triangle} A B C$ satisfy the following equation:

$$
\cos \delta=\sin \alpha \sin \beta \cosh c-\cos \alpha \cos \beta
$$

Proof. Evaluate the first term of the right-hand side of (99) by making use of (43a), (43b), and (43c):

$$
\begin{aligned}
& \sin \alpha \sin \beta \cosh c= \frac{1}{r_{a} r_{b}} O_{c a} O_{c b}\left(1-\cos c_{1} \cos c_{2}\right) \\
&= \frac{1}{r_{a} r_{b}} O_{c a} O_{c b} \\
& \times\left(1-\frac{O_{c b}^{2}-r_{b}^{2}+r_{c}^{2}}{2 r_{c} O_{c b}} \frac{O_{c a}^{2}-r_{a}^{2}+r_{c}^{2}}{2 r_{c} O_{a c}}\right) \\
&= \frac{1}{r_{a} r_{b}} O_{a c} O_{c b}-\frac{1}{r_{a} r_{b}} O_{a c} O_{c b} \\
& \times\left(\frac{O_{c b}^{2}-r_{b}^{2}+r_{c}^{2}}{2 r_{c} O_{c b}} \frac{O_{a c}^{2}-r_{a}^{2}+r_{c}^{2}}{2 r_{c} O_{a c}}\right) \\
&= \frac{1}{4 r_{a} r_{b} r_{c}^{2}}\left(4 O_{a c} O_{c b} r_{c}^{2}-O_{c b}^{2} O_{c a}^{2}\right. \\
&\left.\quad-\left(O_{c b}^{2}+O_{c a}^{2}\right) r_{c}^{2}+O_{c b}^{2} r_{a}^{2}+O_{a c}^{2} r_{b}^{2}\right) .
\end{aligned}
$$

Now calculate the product $\cos \alpha \cos \beta$ by using the following formulae:

$$
\begin{aligned}
& \cos \alpha=\frac{1}{2 r_{b} r_{c}}\left(r_{c}^{2}+r_{b}^{2}-O_{c b}^{2}\right), \\
& \cos \beta=-\frac{1}{2 r_{a} r_{c}}\left(r_{c}^{2}+r_{a}^{2}-O_{a c}^{2}\right) .
\end{aligned}
$$

We obtain

$$
\begin{aligned}
\cos \alpha \cos \beta= & \frac{1}{2 r_{b} r_{c}}\left(r_{c}^{2}+r_{b}^{2}-O_{c b}^{2}\right) \frac{1}{2 r_{a} r_{c}}\left(r_{c}^{2}+r_{a}^{2}-O_{a c}^{2}\right) \\
= & \frac{1}{4 r_{b} r_{a} r_{c}^{2}} \\
& \times\left(O_{c b}^{2} O_{a c}^{2}-\left(O_{c b}^{2}+O_{a c}^{2}\right) r_{c}^{2}-O_{c b}^{2} r_{a}^{2}-O_{a c}^{2} r_{b}^{2}\right) .
\end{aligned}
$$

By using (100) and (102) calculate the difference

$$
\begin{aligned}
& \sin \alpha \sin \beta \cosh c-\cos \alpha \cos \beta \\
& =\frac{1}{4 r_{a} r_{b} r_{c}^{2}}\left(4 O_{a c} O_{c b} r_{c}^{2}-O_{c b}^{2} O_{a c}^{2}-\left(O_{c b}^{2}+O_{a c}^{2}\right) r_{c}^{2}\right. \\
& \left.\quad+O_{c b}^{2} r_{a}^{2}+O_{a c}^{2} r_{b}^{2}\right)-\frac{1}{4 r_{b} r_{a} r_{c}^{2}} \\
& \quad \times\left(+O_{c b}^{2} O_{a c}^{2}-\left(O_{c b}^{2}+O_{a c}^{2}\right) r_{c}^{2}-O_{c b}^{2} r_{a}^{2}-O_{a c}^{2} r_{b}^{2}\right) \\
& =\frac{1}{2 r_{b} r_{a}}\left(r_{a}^{2}+r_{b}^{2}-O_{a b}^{2}\right)=\cos \delta .
\end{aligned}
$$

Thus, we got (99).

The other two equations, (80) and (81), are proved analogously.

\section{Concluding Remarks}

We have proved three hyperbolic cosine-sine theorems for the triangular formed by arcs of three intersecting semicircles by using only elements of the Euclidean geometry. This geometrical figure is one of the basic figures of the Poincaré model of hyperbolic geometry. Conventionally, in the textbooks on the Poincaré model of hyperbolic geometry these theorems are proved by employing invariance properties of the cross-ratio with respect to Möbius group transformations. In that case one remains with the impression that the hyperbolic cosinesine theorems are consequences of a special structure of the $H$ half-plane. We have shown that the hyperbolic trigonometry as well as periodic trigonometry arises on Euclidean plane in a natural way.

The method of parametrization of the mass-shell equation via the hyperbolic angle, the rapidity, is widely used in the relativistic physics [4]. The quadratic equation (3) is closely related to the mass-shell equation

$$
p_{0}^{2}-p^{2}=m^{2} c^{2}
$$

where $m c$ plays the role of radius of the semicircle $2 m c=x_{2}-$ $x_{1}$. Within the framework of the present method we come to the following parametrization of energy momentum of the relativistic particle [11]:

$$
p_{0}=m c \operatorname{coth}(m c \phi), \quad p=\frac{m c}{\sinh (m c \phi)}, \quad 0 \leq \phi<\infty .
$$


In this way, the method developed in this paper may be used in order to give a new geometrical interpretation of rapidity widely used in relativistic physics (see, e.g., [12] and references therein).

\section{References}

[1] S. Stahl, The Poincaré Half-Plane, Jones and Bartlett, Boston, Mass, USA, 1993.

[2] J. W. Anderson, Hyperbolic Geometry, Springer, London, UK, 1999.

[3] S. Katok, Fuchsian Groups, Chicago Lectures in Mathematics, University of Chicago Press, Chicago, Ill, USA, 1992.

[4] A. A. Ungar, "Hyperbolic trigonometry and its application in the Poincaré ball model of hyperbolic geometry," Computers \& Mathematics with Applications, vol. 41, no. 1-2, pp. 135-147, 2001.

[5] R. M. Yamaleev, "Multicomplex algebras on polynomials and generalized Hamilton dynamics," Journal of Mathematical Analysis and Applications, vol. 322, no. 2, pp. 815-824, 2006.

[6] R. M. Yamaleev, "Geometrical interpretation of general complex algebra and its application in relativistic mechanics," Advances in Applied Clifford Algebras, vol. 17, no. 2, pp. 281-305, 2007.

[7] V. F. Kagan, Foundations of Geometry. Part I., Gostekhizdat, Moscow, Russia, 1949.

[8] N. J. Lobatschewsky, "Etudes géométriques sur la théorie de parallèles, suivis d'un extrait de la correspondance de Gauss et de Schumacher," in Mémoires de la Société des sciences physiques et naturelles de Bordeaux, G. J. Hoäuel, Ed., vol. 4, Société des sciences physiques et naturelles de Bordeaux, Bordeaux, France, 1866.

[9] G. Dattoli and M. Del Franco, "Hyperbolic and circular trigonometryand application to special relativity," In press, http://arxiv.org/abs/1002.4728 .

[10] R. M. Yamaleev, "Hyperbolic cosines and sinestheorem for the triangle formed by intersections of three semicircles on Euclidean plane," In press, http://arxiv.org/abs/1104.5135 .

[11] R. M. Yamaleev, "New representation for energy-momentum and its applications to relativistic dynamics," Physics of Atomic Nuclei, vol. 74, no. 12, pp. 1775-1782, 2011.

[12] N. A. Chernikov, "Lobachevsky geometry and relativistic mechanics," Physics of Atomic Nuclei, vol. 4, no. 3, pp. 774-809, 1973. 


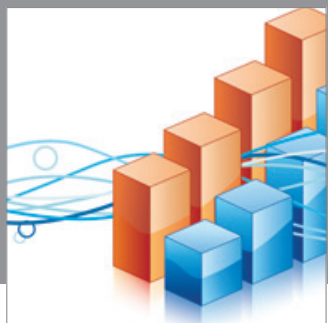

Advances in

Operations Research

mansans

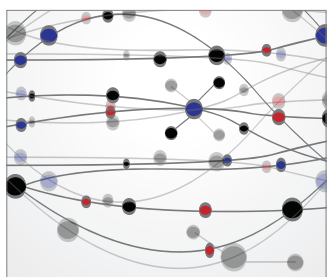

The Scientific World Journal
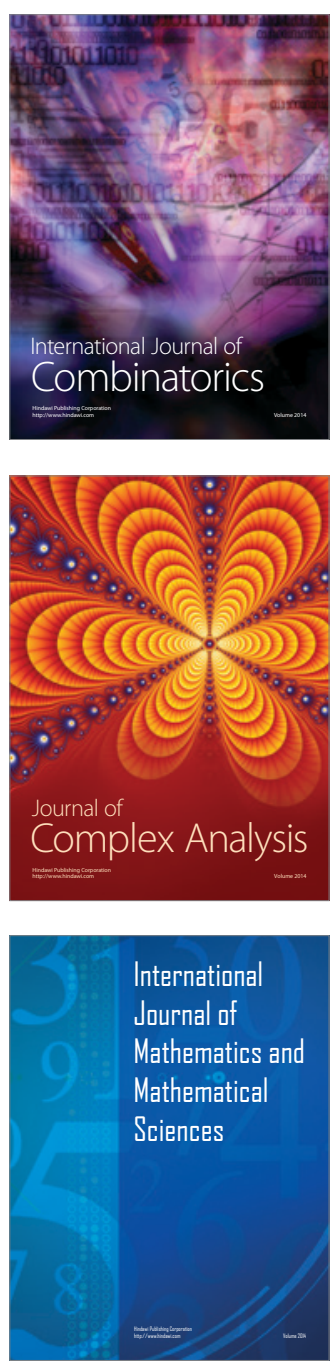
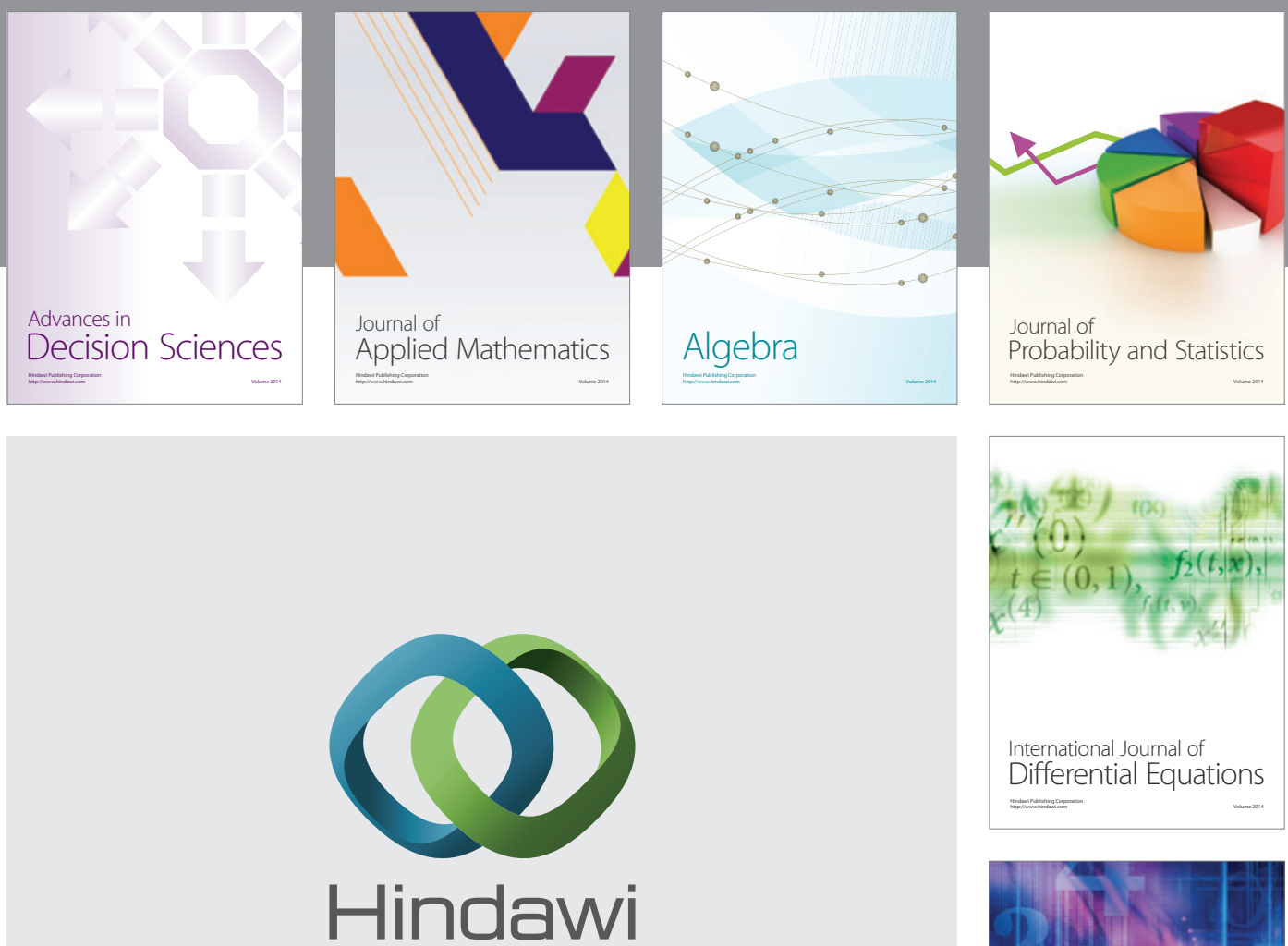

Submit your manuscripts at http://www.hindawi.com
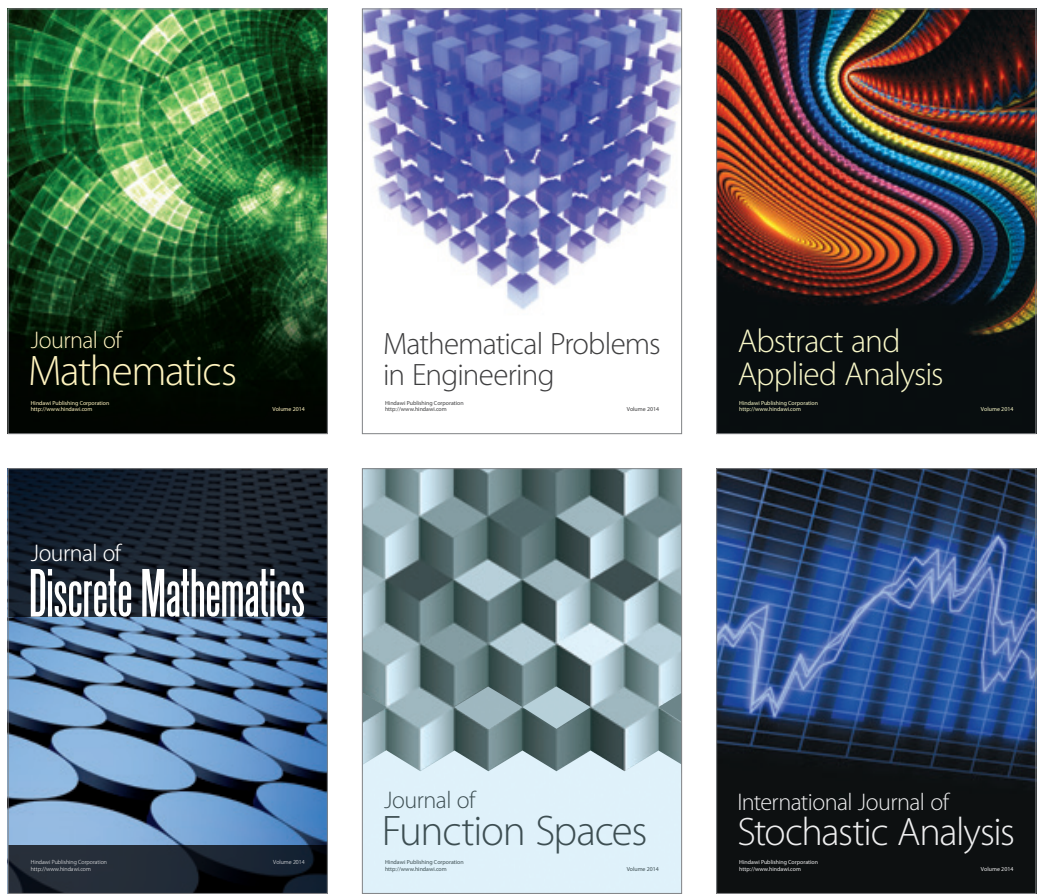

Journal of

Function Spaces

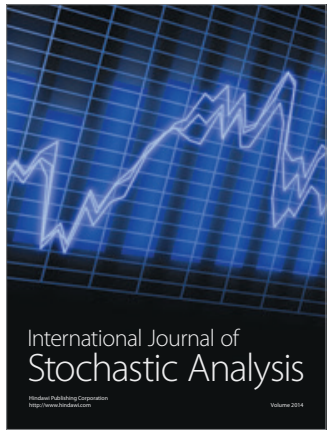

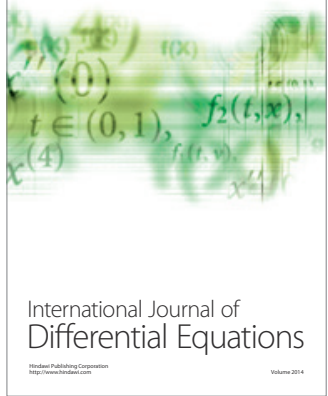
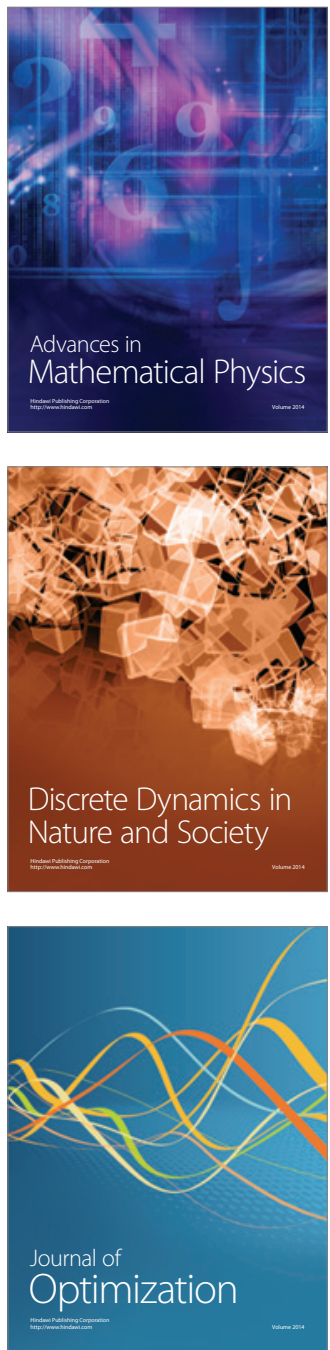